\title{
Desempenho dos Índices de Gravidade na Predição de Complicações Pós-Operatórias de Revascularização Miocárdica
}

\author{
Performance of Severity Indices to Estimate Postoperative Complications of Myocardial Revascularization \\ Silvana Alves dos Santos Franzotti, ${ }^{10}$ Dyenily Alessi Sloboda, ${ }^{1}$ Juliana Rosendo Silva, ${ }^{2}$ Ellian Amorim Santos \\ Souza, ${ }^{1}$ Jessica Zamora Reboreda, ${ }^{1}$ Renata Eloah de Lucena Ferretti-Rebustini, ${ }^{1}$ Lilia de Souza Nogueira ${ }^{10}$ \\ Universidade de São Paulo - Escola de Enfermagem, ${ }^{1}$ São Paulo, SP - Brasil \\ Universidade de São Paulo Faculdade de Medicina Hospital das Clínicas Instituto do Coração, ${ }^{2}$ São Paulo, SP - Brasil
}

\section{Resumo}

Fundamento: Os pacientes em pós-operatório (PO) de cirurgia de revascularização miocárdica (CRM) internados em unidade de terapia intensiva (UTI) apresentam risco de complicações que aumentam o tempo de permanência e a morbimortalidade. Portanto, é fundamental o reconhecimento precoce desses riscos para otimizar estratégias de prevenção e desfecho clínico satisfatório.

Objetivo: Analisar o desempenho de índices de gravidade na predição de complicações em pacientes no PO de CRM durante a permanência na UTI.

Métodos: Estudo transversal, com análise retrospectiva de prontuários eletrônicos de pacientes com idade $\geq 18$ anos submetidos à CRM isolada e admitidos na UTI de um hospital cardiológico, em São Paulo, Brasil. As áreas sob as curvas receiver operating characteristic (AUC) com intervalo de confiança de 95\% foram analisadas para verificar a acurácia dos índices European System for Cardiac Operative Risk Evaluation (EuroScore), Acute Physiology and Chronic Health Evaluation (APACHE II), Simplified Acute Physiology Score (SAPS II) e Sequential Organ Failure Assessment (SOFA) na predição de complicações.

Resultados: A casuística foi composta por 366 pacientes $(64,58 \pm 9,42$ anos; $75,96 \%$ sexo masculino). As complicações identificadas foram respiratórias $(\mathbf{2 4 , 3 2 \% )}$, cardiológicas (19,95\%), neurológicas (10,38\%), hematológicas (10,38\%), infecciosas $(6,56 \%)$ e renais (3,55\%). O APACHE II apresentou satisfatório desempenho para a predição de complicações neurológicas (AUC 0,72) e renais (AUC 0,78).

Conclusão: O APACHE II se destacou na previsão das complicações neurológicas e renais. Nenhum dos índices teve bom desempenho na predição das outras complicações analisadas. Portanto, os índices de gravidade não devem ser utilizados indiscriminadamente com o objetivo de predizer todas as complicações frequentemente apresentadas por pacientes após CRM. (Arq Bras Cardiol. 2020; 115(3):452-459)

Palavras-chave: Doenças Cardiovasculares/complicações; Doenças Cardiovasculares/cirurgia; Revascularização Miocárdica/complicações; Cuidados Pós-Operatórios; Complicações Pós-Operatórias; Morbimortalidade; Indicadores de Gravidade de Doença; Unidade de Terapia Intensiva.

\footnotetext{
Abstract

Background: Patients in the postoperative period of myocardial revascularization (Coronary Artery Bypass Grafting - CABG) surgery admitted to the intensive care unit (ICU) are at risk of complications which increase the length of stay and morbidity and mortality. Therefore, early recognition of these risks is essential to optimize prevention strategies and a satisfactory clinical outcome.
}

Objective: To analyze the performance of severity indices in predicting complications in patients in the postoperative of CABC during the ICU stay. Methods: A cross-sectional study with retrospective analysis of electronic medical records of patients aged $\geq 18$ years who underwent isolated CABG and were admitted to the ICU of a cardiology hospital in São Paulo, Brazil. The areas under the receiver operating characteristic curves (AUC) with a 95\% confidence interval were analyzed to verify the accuracy of the European System for Cardiac Operative Risk Evaluation (EuroScore), Acute Physiology and Chronic Health Evaluation (APACHE II), Simplified Acute Physiology Score (SAPS II) and Sequential Organ Failure Assessment (SOFA) indices in predicting complications.

Correspondência: Silvana Alves dos Santos Franzotti •

Universidade de São Paulo - Escola de Enfermagem - Av. Dr. Eneas de Carvalho Aguiar, 419. CEP 05403-000, São Paulo, SP - Brasil E-mail: silvana_alves90@hotmail.com

Artigo recebido em 19/02/2019, revisado em 24/07/2019, aceito em 10/09/2019

DOI: https://doi.org/10.36660/abc.20190120 
Results: The sample consisted of 366 patients ( $64.58 \pm 9.42$ years; $75.96 \%$ male). The complications identified were: respiratory (24.32\%), cardiovascular (19.95\%), neurological (10.38\%), hematological (10.38\%), infectious (6.56\%) and renal (3.55\%). APACHE II showed satisfactory performance for predicting neurological (AUC 0.72) and renal (AUC 0.78) complications.

Conclusion: APACHE II excelled in predicting neurological and renal complications. None of the indices performed well in predicting the other analyzed complications. Therefore, severity indices should not be used indiscriminately in order to predict all complications frequently presented by patients after CABG. (Arq Bras Cardiol. 2020; 115(3):452-459)

Keywords: Cardiovascular Diseases/complications; Cardiovascular Diseases/surgery; Myocardial Revascularization/complications; Postoperative Care; Indicators of Morbidity and Mortality; Severity of IIIness Index; Intensive Care Units.

Full texts in English - http://www.arquivosonline.com.br

\section{Introdução}

As doenças cardiovasculares são responsáveis por elevada taxa de mortalidade no Brasil e no mundo, com destaque à alta prevalência do infarto agudo do miocárdio (IAM)..$^{1-3}$ Dentre as principais terapias voltadas para o tratamento do IAM, aponta-se a cirurgia de revascularização miocárdica (CRM). 4,5

Resultados bem-sucedidos da CRM dependem de um excelente cuidado pós-operatório (PO) realizado na unidade de terapia intensiva (UTI), uma vez que os pacientes estão expostos a inúmeros efeitos adversos decorrentes da complexa resposta inflamatória sistêmica orgânica, apresentam instabilidade hemodinâmica e maior risco de desenvolver complicações durante o tratamento intensivo. ${ }^{6-9}$

Reconhecer precocemente os pacientes que apresentam risco elevado de complicações é essencial para otimizar o tratamento, aumentar a chance de desfecho satisfatório, ${ }^{6-9}$ além de reduzir custos, uma vez a ocorrência destes eventos após CRM aumenta significativamente os gastos com tratamento e hospitalização. ${ }^{10}$

Estudos recentes têm mostrado a excelente acurácia de diferentes índices de gravidade na predição de mortalidade de pacientes no $\mathrm{PO}$ de cirurgia cardíaca na UTI. ${ }^{11-13} \mathrm{Em}$ relação à ocorrência de complicações, pesquisadores identificaram que o índice European System for Cardiac Operative Risk Evaluation (EuroScore) foi bom preditor de falência respiratória e lesão renal aguda dialítica em pacientes após cirurgia cardíaca. ${ }^{14}$

A partir do exposto, até o presente momento, não existem índices específicos capazes de prever satisfatoriamente o risco de um paciente desenvolver diferentes complicações na UTI após CRM. Além disso, na prática clínica, os profissionais intensivistas identificam estreita relação entre gravidade do paciente no PO de CRM e ocorrência de complicação.

Neste sentido, reforça-se a importância de analisar e investigar o desempenho de diferentes índices de gravidade na predição dessas complicações uma vez que essas informações poderão auxiliar em estratégias de tratamento, melhorar a segurança e a qualidade da assistência prestada ao paciente no PO de CRM e, consequentemente, o desfecho clínico. Sendo assim, o presente estudo tem como objetivo analisar o desempenho de índices de gravidade na predição de complicações em pacientes no PO de revascularização miocárdica durante a permanência na UTI.

\section{Métodos}

Estudo analítico transversal, com dados coletados retrospectivamente por meio da análise de prontuários eletrônicos de pacientes admitidos na UTI cirúrgica de uma instituição especializada em cardiopneumologia de alta complexidade, localizada em São Paulo, Brasil. A pesquisa foi aprovada pelo Comitê de Ética da instituição (Parecer no 2.831.457).

\section{Casuística}

A casuística, por conveniência, foi composta por pacientes submetidos à CRM entre agosto de 2014 a julho de 2015 e que atenderam aos seguintes critérios de inclusão: idade $\geq 18$ anos e ser admitido na UTI diretamente do centro cirúrgico após CRM isolada. Optou-se por incluir pacientes submetidos à CRM exclusiva para evitar a interferência de outros procedimentos cirúrgicos nos desfechos clínicos analisados.

\section{Variáveis Analisadas}

Para atender ao propósito do estudo, foram extraídas dos prontuários dos pacientes as variáveis relacionadas aos dados demográficos (idade, sexo e etnia), à presença dos fatores de risco para doença arterial coronariana (hipertensão arterial sistêmica, diabetes mellitus e dislipidemia) e de IAM prévio, ao procedimento cirúrgico realizado [tipo de cirurgia (eletiva ou de urgência), uso de circulação extracorpórea (CEC), tempo de CEC, tipo de enxerto implantado (arterial, venoso ou misto, ou seja, arterial e venoso) e quantidade média de enxertos recebidos] e à internação na unidade crítica [ocorrência ou não das complicações cardiológicas (arritmia, choque cardiogênico, derrame pericárdico e pericardite), respiratórias (congestão pulmonar, derrame pleural, atelectasia e pneumotórax), neurológicas (delirium, acidente vascular encefálico e crise convulsiva), infecciosas (infecção de ferida operatória, mediastinite, pneumonia, infecção da corrente sanguínea), renais (lesão renal aguda com ou sem terapia de substituição renal, lesão renal crônica agudizada) e hematológicas (sangramento, distúrbios de coagulação, necessidade de transfusão sanguínea), além do tempo de permanência na UTI em dias e da condição de saída da unidade crítica (sobrevivente ou não sobrevivente)]. As complicações analisadas foram selecionadas no estudo por serem as mais frequentes, segundo dados da literatura. $6,8,9$ Ressalta-se que as complicações analisadas na pesquisa foram consideradas presentes a partir do diagnóstico médico das mesmas registrado nos prontuários dos pacientes.

Além dessas variáveis, foram coletadas as informações necessárias para o cálculo dos índices EuroScore, ${ }^{15}$ Acute Physiology and Chronic Health Evaluation (APACHE II), ${ }^{16}$ Simplified Acute Physiology Score (SAPS II) ${ }^{17}$ e Sepsis- 
related Organ Failure Assessment (SOFA). ${ }^{18} \mathrm{~A}$ escolha por esses índices no presente estudo foi pautada na facilidade do cálculo e maior frequência de utilização dos mesmos em estudos ${ }^{10-14}$ que analisam exclusivamente pacientes submetidos à cirurgia cardíaca.

\section{Procedimento de Coleta dos Dados}

Para a coleta de dados, inicialmente foi elaborada uma lista de pacientes submetidos à CRM isolada a partir das descrições cirúrgicas contidas no sistema informatizado da instituição. A partir desta lista, observou-se que todos os pacientes tinham idade superior a 18 anos e foram admitidos na UTI diretamente do centro cirúrgico, não havendo exclusões posteriores.

Para identificar as possíveis complicações ocorridas durante a internação do paciente na UTI após CRM, foram avaliadas todas as evoluções diárias da equipe multiprofissional.

Os dados necessários para o cálculo do risco de morte segundo o EuroScore foram obtidos a partir de informações dos pacientes referentes ao período pré-operatório. Para o cálculo dos índices APACHE II, SAPS II e SOFA foram analisados os sinais vitais e exames laboratoriais das primeiras 24 horas de internação do paciente na UTI, sendo considerados os piores valores, ou seja, que mais pontuavam no índice.

Ressalta-se que a equipe de coleta de dados, formada por enfermeiras que cursavam residência em enfermagem na instituição, foi capacitada por meio de treinamentos in loco. Inicialmente, a coleta de dados foi realizada em pares e qualquer discordância no resgate das informações era prontamente corrigida. Tal processo foi realizado até que se atingisse a uniformidade na coleta de dados entre as enfermeiras.

\section{Análise Estatística}

Para a caracterização da amostra, foram calculadas médias e desvios-padrão para as variáveis contínuas e percentagens absolutas e relativas para as categóricas. Na descrição dos índices de gravidade, média, desvio-padrão, mediana e valores mínimos e máximos foram identificados.

Para avaliar o desempenho dos índices de gravidade na predição das diferentes complicações ocorridas nos pacientes na UTI foram identificados valores de área sob a curva receiver operating characteristic (AUC) com respectivo intervalo de confiança (IC) e nível de significância de 95\%, ponto de corte ideal calculado pelo método de Youden, sensibilidade, especificidade, valor preditivo positivo (VPP), valor preditivo negativo (VPN) e acurácia. Uma estimativa pontual de AUC $\geq 0,70$ foi definida como desempenho satisfatório do índice para o desfecho analisado. O software $\mathrm{R}$ versão 3.6.0 para Windows foi utilizado para a análise dos dados.

\section{Resultados}

A casuística foi composta por 366 pacientes, com idade média de 64,58 $( \pm 9,42)$ anos, predomínio do sexo masculino $(75,96 \%)$ e etnia caucasiana $(88,25 \%)$. A presença de hipertensão arterial sistêmica (74,59\%) se destacou em relação à dislipidemia $(54,10 \%)$ e diabetes mellitus $(48,63 \%)$ como fatores de risco para doença arterial coronariana.

Dos 366 pacientes analisados, aproximadamente metade ( $n=180 ; 49,18 \%$ ) apresentava IAM prévio e a CRM eletiva $(60,38 \%)$ prevaleceu na amostra. Um total de 295 pacientes $(81,69 \%)$ foi submetido à CEC durante o procedimento cirúrgico com tempo médio de CEC de 90,69 ( \pm 25,69) minutos. O principal tipo de enxerto implantado foi o misto $(80,60 \%)$ seguido do arterial $(12,84 \%)$ e, em média, os pacientes receberam $2,60( \pm 0,87)$ enxertos.

A Tabela 1 apresenta os dados descritivos dos índices de gravidade aplicados na casuística.

O maior risco de morte estimado pelo EuroScore foi de $29,29 \%$. Um total de 9 pacientes tinha chance de morte na UTI acima de 50\% conforme previsto pelo APACHE II, 2 segundo SOFA e 1 de acordo com o SAPS II.

$\mathrm{Na}$ amostra de 366 pacientes, as frequências das complicações evidenciadas no PO de CRM na UTI foram: respiratórias (24,32\%), cardiológicas (19,95\%), neurológicas $(10,38 \%)$, hematológicas $(10,38 \%)$, infecciosas $(6,56 \%)$ e renais $(3,55 \%)$.

Entre elas, destacaram-se a congestão pulmonar $(n=52)$ e o derrame pleural $(n=30)$ para complicações respiratórias, arritmias $(n=55)$ nas cardiológicas, delirium $(n=34)$ nas neurológicas e pneumonia $(n=14)$ para as infecciosas. A lesão renal aguda e a hemorragia representaram a totalidade das complicações renais e hematológicas, respectivamente.

O tempo de permanência dos pacientes na UTI foi 4,64 $( \pm 5,64)$ dias e todos os doentes que evoluíram a óbito na unidade crítica $(n=19 ; 5,20 \%)$ apresentaram algum tipo de complicação no PO de CRM.

A partir dos dados da Figura 1 e Tabela 2, identificou-se que o APACHE II foi o índice que apresentou melhor desempenho na predição de complicações neurológicas e renais apresentadas

Tabela 1 - Estatística descritiva de índices de gravidade calculados em pacientes submetidos à CRM

\begin{tabular}{lllll}
\hline Índice de gravidade & Média & Desvio padrão & Mediana & Mín-Máx \\
EuroScore* & 4,08 & 4,70 & 2,50 & $0,88-29,29$ \\
SOFA† & 5,84 & 1,88 & 6,00 & $2,00-13,00$ \\
APACHE II & 14,21 & 4,96 & 14,00 & $4,00-41,00$ \\
SAPS II§ & 24,37 & 8,66 & 24,00 & $7,00-54,00$ \\
\hline
\end{tabular}

*EuroScore: European System for Cardiac Operative Risk Evaluation; TSOFA: Sepsis-related Organ Failure Assessment; $¥$ APACHE II: Acute Physiology and Chronic Health Evaluation; §SAPS II: Simplified Acute Physiology Score. 
Complicações neurológicas

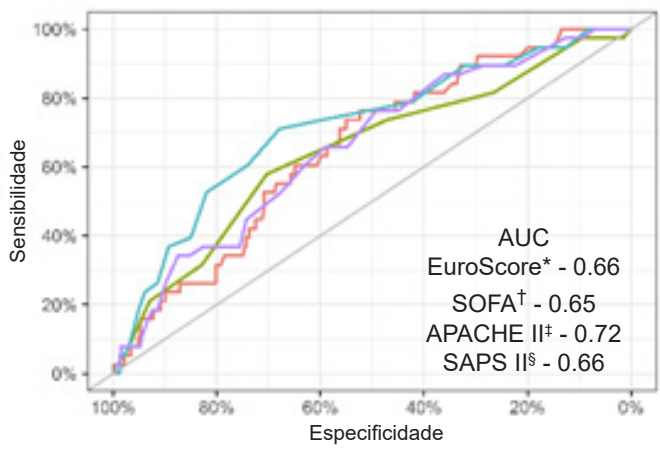

Complicações infecciosas

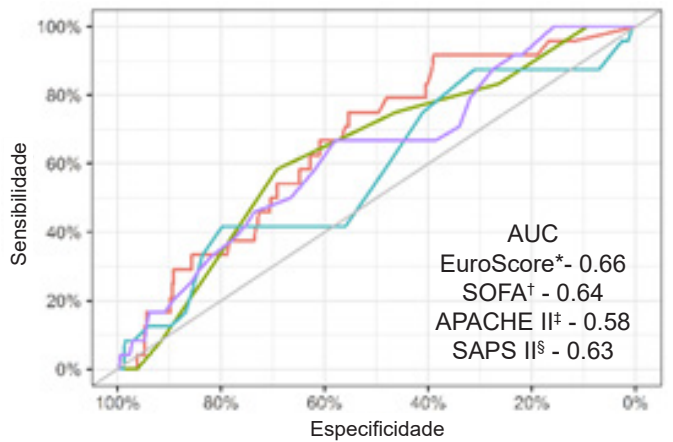

Complicações renais

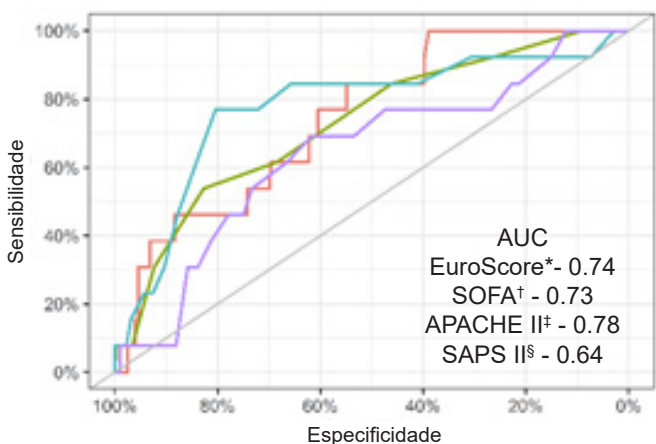

Complicações cardiológicas

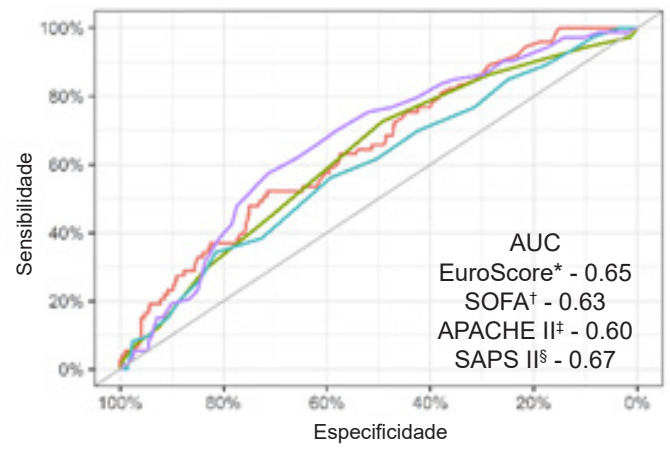

- Euroscore

- SOFA

- APACHE II

- SAPS II
- Euroscore

- SOFA

- APACHE II

- SAPS II

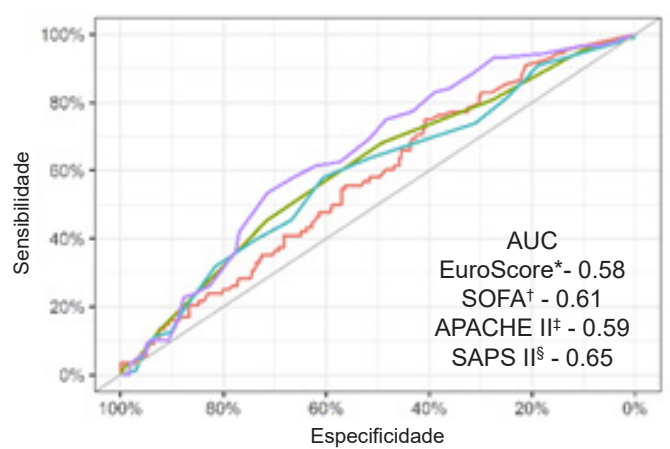

- Euroscore

- SOFA

- APACHE II

- SAPS II

Figura 1 - Receiver Operating Characteristic Curves dos indices de gravidade na predição de complicações em pacientes após CRM. *EuroScore: European

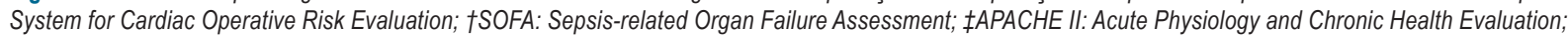
§SAPS II: Simplified Acute Physiology Score. 


\section{Artigo Original}

Tabela 2 - Desempenho dos índices de gravidade na predição de complicações em pacientes no pós-operatório de CRM na UTI

\begin{tabular}{|c|c|c|c|c|c|c|c|c|}
\hline Neurológicas & AUC" & $\begin{array}{l}\text { IC95\%" } \\
\text { mín-máx }\end{array}$ & $\begin{array}{l}\text { Ponto de } \\
\text { corte }\end{array}$ & Sensibilidade & Especificidade & VPP\# & $\mathrm{VPN}^{* *}$ & Acurácia \\
\hline EuroScore* & 0,66 & $0,57-0,74$ & 2,59 & 73,68 & 55,05 & 16,00 & 94,74 & 56,99 \\
\hline SOFA $^{\dagger}$ & 0,65 & $0,55-0,74$ & 6,50 & 57,89 & 70,34 & 18,49 & 93,50 & 69,04 \\
\hline APACHE $\|^{\ddagger}$ & 0,72 & $0,63-0,81$ & 15,50 & 71,05 & 67,89 & 20,45 & 95,28 & 68,22 \\
\hline SAPS $\|^{\S}$ & 0,66 & $0,57-0,75$ & 23,50 & 76,32 & 49,24 & 14,87 & 94,71 & 52,05 \\
\hline Cardiológicas & $\mathrm{AUC}^{\prime \prime}$ & $\begin{array}{l}\text { IC95\%" } \\
\text { mín-máx }\end{array}$ & $\begin{array}{l}\text { Ponto de } \\
\text { corte }\end{array}$ & Sensibilidade & Especificidade & VPP\# & $\mathrm{VPN}^{* *}$ & Acurácia \\
\hline EuroScore ${ }^{*}$ & 0,65 & $0,58-0,72$ & 3,50 & 52,05 & 71,58 & 31,40 & 85,66 & 67,67 \\
\hline SOFA $^{\dagger}$ & 0,63 & $0,56-0,70$ & 5,50 & 72,60 & 49,32 & 26,37 & 87,80 & 53,97 \\
\hline APACHE $\|^{\ddagger}$ & 0,60 & $0,52-0,67$ & 17,50 & 34,25 & 81,51 & 31,65 & 83,22 & 72,05 \\
\hline SAPS $\|^{\S}$ & 0,67 & $0,60-0,74$ & 27,50 & 57,53 & 71,23 & 33,33 & 87,03 & 68,49 \\
\hline Infecciosas & AUC" & $\begin{array}{l}\text { IC95\%" } \\
\text { mín-máx }\end{array}$ & $\begin{array}{l}\text { Ponto de } \\
\text { corte }\end{array}$ & Sensibilidade & Especificidade & VPP\# & $\mathrm{VPN}^{* *}$ & Acurácia \\
\hline EuroScore* & 0,66 & $0,56-0,78$ & 1,92 & 91,67 & 39,00 & 9,57 & 98,52 & 42,47 \\
\hline SOFA $^{\dagger}$ & 0,64 & $0,52-0,74$ & 6,50 & 58,33 & 69,21 & 11,76 & 95,93 & 68,49 \\
\hline APACHE $\|^{\ddagger}$ & 0,58 & $0,46-0,70$ & 17,50 & 41,67 & 79,77 & 12,66 & 95,10 & 77,26 \\
\hline SAPS $\|^{\S}$ & 0,63 & $0,53-0,74$ & 25,50 & 66,67 & 58,06 & 10,06 & 96,12 & 58,63 \\
\hline Respiratórias & AUC" & $\begin{array}{l}\text { IC95\%" } \\
\text { mín-máx }\end{array}$ & $\begin{array}{l}\text { Ponto de } \\
\text { corte }\end{array}$ & Sensibilidade & Especificidade & VPP\# & $\mathrm{VPN}^{* *}$ & Acurácia \\
\hline EuroScore* & 0,58 & $0,51-0,64$ & 1,92 & 75,00 & 40,79 & 28,70 & 83,70 & 49,04 \\
\hline SOFA $^{\dagger}$ & 0,61 & $0,54-0,67$ & 5,50 & 68,18 & 49,10 & 29,85 & 82,93 & 53,70 \\
\hline APACHE $\|^{\ddagger}$ & 0,59 & $0,52-0,66$ & 14,50 & 57,95 & 60,65 & 31,87 & 81,95 & 60,00 \\
\hline SAPS $\|^{\S}$ & 0,65 & $0,58-0,71$ & 27,50 & 53,41 & 71,48 & 37,30 & 82,85 & 67,12 \\
\hline Renais & $\mathrm{AUC}^{\prime \prime}$ & $\begin{array}{l}\text { IC95\%" } \\
\text { mín-máx }\end{array}$ & $\begin{array}{l}\text { Ponto de } \\
\text { corte }\end{array}$ & Sensibilidade & Especificidade & VPP\# & VPN $^{* *}$ & Acurácia \\
\hline EuroScore* & 0,74 & $0,62-0,86$ & 2,65 & 84,62 & 54,83 & 6,47 & 98,97 & 55,89 \\
\hline $\mathrm{SOFA}^{\dagger}$ & 0,73 & $0,59-0,87$ & 7,50 & 53,85 & 82,67 & 10,29 & 97,98 & 81,64 \\
\hline APACHE $\|^{\ddagger}$ & 0,78 & $0,63-0,93$ & 17,50 & 76,92 & 80,40 & 12,66 & 98,95 & 80,27 \\
\hline SAPS $\|^{\S}$ & 0,64 & $0,48-0,80$ & 26,50 & 69,23 & 61,65 & 6,25 & 98,19 & 61,92 \\
\hline Hematológicas & AUC" & $\begin{array}{l}\text { IC95\%匹 } \\
\text { mín-máx }\end{array}$ & $\begin{array}{l}\text { Ponto de } \\
\text { corte }\end{array}$ & Sensibilidade & Especificidade & VPP\# & $\mathrm{VPN}^{* *}$ & Acurácia \\
\hline EuroScore* & 0,57 & $0,48-0,67$ & 1,52 & 71,87 & 47,37 & 92,16 & 16,36 & 69,32 \\
\hline SOFA $^{\dagger}$ & 0,57 & $0,47-0,67$ & 6,50 & 44,74 & 68,81 & 14,29 & 91,46 & 66,30 \\
\hline APACHE $\|^{\ddagger}$ & 0,55 & $0,45-0,65$ & 14,50 & 52,63 & 57,19 & 12,50 & 91,22 & 56,71 \\
\hline SAPS $\|^{\S}$ & 0,60 & $0,50-0,68$ & 21,50 & 76,32 & 40,06 & 12,89 & 93,57 & 43,84 \\
\hline
\end{tabular}

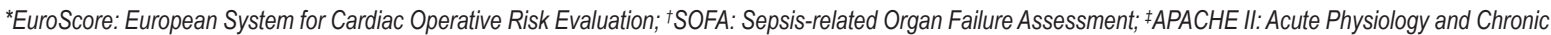
Health Evaluation; SSAPS II: Simplified Acute Physiology Score; "AUC: área sob a curva; "IC: intervalo de confiança; "VPP: valor preditivo positivo; *VPN: valor preditivo negativo.

pelos pacientes no PO de CRM durante a internação na UTI, com maiores valores de AUC e resultados satisfatórios na análise dos valores de sensibilidade, especificidade, VPN e acurácia em comparação aos outros índices. Em contrapartida, nenhum dos índices aplicados (EuroScore, SOFA, APACHE II e SAPS II) apresentou bom desempenho na predição das outras complicações (cardiológicas, infecciosas, respiratórias e hematológicas) investigadas no estudo.

\section{Discussão}

Os dados da casuística deste estudo mostraram predomínio do sexo masculino e idade média aproximada de 64 anos, corroborando com achados na literatura. ${ }^{10,11}$

$\mathrm{Na}$ análise dos índices de gravidade, identificou-se que o valor médio do EuroScore encontrado neste estudo foi superior aos resultados de pesquisas que aplicaram o índice 
em pacientes após cirurgia cardíaca ${ }^{11,14}$ ou CRM isolada. ${ }^{10}$ Entretanto, os valores do SOFA, APACHE II e SAPS II desta investigação foram inferiores aos achados da pesquisa que analisou 150 pacientes submetidos à cirurgia cardíaca e comparou o desempenho desses índices e do Cardiac Surgery Score (CASUS) na predição de desfechos clínicos, com resultado satisfatório para o CASUS. ${ }^{11}$

As principais complicações apresentadas pelos pacientes no período PO da CRM na UTI corroboram com dados da literatura. ${ }^{7-9}$ Distúrbios respiratórios são comuns em pacientes após cirurgia cardíaca, mas poucos necessitam de ventilação mecânica por mais de 72 horas para tratamento. ${ }^{19,20}$ Arritmias supraventriculares, especialmente fibrilação atrial (FA), ocorrem com frequência após cirurgia cardíaca e contribuem para o aumento de tempo de internação hospitalar e risco de acidente vascular encefálico, sendo que a terapia profilática diminui a incidência de FA em 50\%.7

Interessante observar o número de pacientes que apresentaram delirium na presente investigação. Estudos mostram que este evento é um problema significativo após cirurgia cardíaca, com impacto negativo no desfecho clínico dos pacientes, ${ }^{21-23}$ e os principais fatores de risco são uso de benzodiazepínico, restrições e imobilizações no leito devido à presença de dispositivos. ${ }^{24}$ Além disso, deve-se ressaltar que 247 pacientes da amostra tinham mais de 60 anos (idade média aproximada de 64 anos) e revisão da literatura ${ }^{25}$ evidenciou que existe associação entre idade avançada e maior risco de delirium na UTI.

As infecções nosocomiais ocorrem em 10 a $20 \%$ dos pacientes submetidos à cirurgia cardíaca e muitas delas são evitáveis. ${ }^{7}$ Por fim, destacam-se a lesão renal aguda e a hemorragia. A primeira decorre principalmente de hipoperfusão, hemólise e citocinas inflamatórias e, quando é necessária a terapia de substituição renal, há aumento significativo de mortalidade. ${ }^{7,26}$ A hemorragia excessiva, identificada habitualmente pelas características e volume do débito nos drenos de tórax, ocorre principalmente após cirurgias de emergência, uso prolongado da CEC, baixa massa corporal e anemia pré-operatória.

Em relação à aplicabilidade dos índices de gravidade, este estudo identificou que apenas o APACHE II apresentou satisfatório desempenho na predição das complicações neurológicas e renais apresentadas pelos pacientes no pós operatório de CRM durante a internação na UTI. Para os outros desfechos analisados, nenhum índice aplicado na casuística foi considerado bom preditor.

Estudos recentes mostram que o APACHE II é considerado um adequado índice preditivo de complicações neurológicas em pacientes cardíacos comatosos após parada cardiorrespiratória ${ }^{27}$ e uma excelente ferramenta prognóstica para mortalidade em pacientes com lesão renal aguda. ${ }^{28}$ Entretanto, essas pesquisas, apesar de analisarem complicações neurológicas e renais ocorridas em UTI, não abordaram especificamente pacientes submetidos à CRM isolada.

Vale ressaltar que o APACHE II é um índice reprodutível, de fácil aplicação, que fornece a probabilidade de morte do paciente a partir de diferentes informações sobre fatores que influenciam este desfecho, como idade, presença de comorbidades e alterações fisiológicas. Uma limitação deste índice é que alguns pacientes apresentam várias comorbidades e apenas uma delas pode ser selecionada. ${ }^{29}$ No contexto cardiovascular, somente a insuficiência cardíaca congestiva classe IV é valorizada no seu cálculo. ${ }^{16}$

Em relação ao EuroScore, estudo brasileiro mostrou valores de $\mathrm{AUC}>0,70$ na predição de complicações renais e respiratórias após cirurgia cardíaca, não especificamente em pacientes submetidos à CRM isolada. ${ }^{14}$ Neste sentido, deve ser reforçado que o EuroScore é um índice específico para avaliação de risco em diferentes cirurgias cardíacas, incluindo a CRM, e considera, para seu cálculo, dados préoperatórios. ${ }^{15}$ Portanto, supõe-se que estas características do índice tenham influenciado para seu baixo desempenho na predição de complicações dos pacientes após CRM isolada do presente estudo.

O SAPS II, em seu estudo de validação, não incluiu pacientes de cirurgia cardíaca. ${ }^{17}$ Acredita-se que esta seja a principal causa do inadequado desempenho do índice nos desfechos analisados.

Por fim, o SOFA ${ }^{18}$ foi criado para identificar disfunção orgânica, que nem sempre está presente nas primeiras 24 horas de internação na UTI. Neste sentido, resultados de pesquisa sugerem que o índice seja calculado sequencialmente, uma vez que, independentemente do valor inicialmente encontrado, o aumento do escore nas primeiras 48 horas do paciente na unidade crítica prediz uma taxa de mortalidade de, no mínimo, 50\%. ${ }^{30}$ Este fato pode ter interferido nos achados do presente estudo, uma vez que o SOFA foi calculado apenas com dados das primeiras 24 horas do paciente na UTI e foram consideradas as complicações ocorridas durante toda a internação na terapia intensiva.

Finalizando, destaca-se que esta pesquisa foi pioneira na avaliação do desempenho de diferentes índices de gravidade, habitualmente aplicados em doentes críticos, na predição de complicações em uma população específica: pacientes submetidos à CRM isolada.

Este estudo apresenta as seguintes limitações: a análise documental retrospectiva dependente da precisão e qualidade das informações registradas pelos profissionais, a casuística foi limitada e o estudo realizado em um único hospital, centro de referência em cardiologia, trazendo possíveis restrições na generalização dos resultados.

\section{Conclusões}

Este estudo permitiu concluir que o índice APACHE II apresentou satisfatório desempenho na predição de complicações neurológicas e renais apresentadas pelos pacientes submetidos à CRM isolada durante a internação na UTI. Em relação à ocorrência de complicações cardiológicas, infecciosas, respiratórias e hematológicas, nenhum dos índices analisados (EuroScore, SOFA, APACHE II e SAPS II) apresentaram bons resultados preditivos.

Portanto, os índices analisados no estudo não devem ser utilizados indiscriminadamente com o objetivo de predizer de maneira precoce as complicações mais frequentes apresentadas por pacientes após CRM na UTI e sugere- 
se a realização de estudos multicêntricos, com amostras significativas, que proponham a criação de índices específicos para prever essas complicações.

\section{Contribuição dos Autores}

Concepção e desenho da pesquisa: Franzotti SAS, FerrettiRebustini REL, Nogueira LS; Obtenção de dados: Franzotti SAS, Silva JR, Souza EAS, Reboreda JZ; Análise e interpretação dos dados e Análise estatística: Franzotti SAS, Sloboda DA, Silva JR, Souza EAS, Reboreda JZ, Ferretti-Rebustini REL, Nogueira LS; Redação do manuscrito: Sloboda DA, Silva JR, Souza EAS, Reboreda JZ, Nogueira LS; Revisão crítica do manuscrito quanto ao conteúdo intelectual importante: Sloboda DA, Ferretti-Rebustini REL, Nogueira LS.

\section{Referências}

1. World Health Organization. (WHO).Global Status Report on noncommunicable diseases 2014 [Internet].[Access in 2018 Jan 03]. Available from: http://www.who.int/nmh/publications/ncd-statusreport-2014/en/.

2. World Health Organization. (WHO). The top 10 causes of death. The 10 leading causes of death in the world, 2000 and 2015 [Internet]. [Access in 2018 Mar. 13]. Available from: http://www.who.int/mediacentre/factsheets/ fs310/en/.

3. Brasil. Ministério da Saúde. Datasus. Causas externas de morbidade e de mortalidade [Internet].[ Access in 2018 mar. 13]. Available from: http:// tabnet.datasus.gov.br/cgi/tabcgi.exe?sim/cnv/evitb10uf.def

4. Anderson JL, Morrow DA. Acute Myocardial Infarction. N Engl J Med. 2017;376(21):2053-64.

5. Dallan LAO, Jatene FB. Myocardial revascularization in the XXI century. Rev Bras Cir Cardiovasc. 2013;28(1):137-44.

6. Stephens RS, Whitman GJR. Postoperative Critical Care of the Adult Cardiac Surgical Patient. Part I: Routine Postoperative Care. Crit Care Med. 2015;43(7):1477-97.

7. Stephens RS, Whitman GJR. Postoperative Critical Care of the Adult Cardiac Surgical Patient. Part II: Procedure-Specific Considerations, Management of Complications and Quality Improvement. Crit Care Med. 2015;43(9):1995-2014.

8. Biancari F, Ruggieri VG, Perrotti A, Svenarud P, Dalén M, Onorati F, et al. European Multicenter Study on Coronary Artery Bypass Grafting (E-CABG registry): Study Protocol for a Prospective Clinical Registry and Proposal of Classification of Postoperative Complications. J Cardiothorac Surg. 2015;10(90):1-12

9. Montrief T, Koyfman A; Long B. Coronary artery bypass graft surgery complications: A review for emergency clinicians. Am J Emerg Med. 2018;36(12):2289-97.

10. Barbosa JL, Thiers CA, Silva AFR, Vianna MM, Gedeon POPR, Martins Neto $\mathrm{L}$, et al. Impact of complications of myocardial revascularization surgery on expenses during hospital stay. Int J Cardiovasc Sci. 2019;32(1):28-34.

11. Exarchopoulos T, Charitidou E, Dedeilias P, Charitos C, Routsi C. Scoring systems for outcome prediction in a cardiac surgical intensive care unit: a comparative study. Am J Crit Care. 2015;24(4):327-34.

12. Chang CH, Chen SW, Fan PC, Lee CC, Yang HY, Chang SW, et al. Sequential organ failure assessment score predicts mortality after coronary artery bypass grafting. BMC Surg. 2017;17(1):22-9

\section{Potencial Conflito de Interesses}

Declaro não haver conflito de interesses pertinentes.

\section{Fontes de Financiamento}

O presente estudo não teve fontes de financiamento externas.

\section{Vinculação Acadêmica}

Não há vinculação deste estudo a programas de pósgraduação.

\section{Aprovação Ética e Consentimento Informado}

Este estudo foi aprovado pelo Comitê de Ética da Escola de Enfermagem da USP sob o número de protocolo 2.831.457. Todos os procedimentos envolvidos nesse estudo estão de acordo com a Declaração de Helsinki de 1975, atualizada em 2013.

13. Jentzer J, Bennett C, Wiley BM, Murphree DH, Keegan MT, Gajic O. Predictive value of the Sequential Organ Failure Assessment Score for mortality in a contemporary cardiac intensive care unit population. J Am Heart Assoc. 2018;7(6):1-11

14. Andrade ING, Moraes Neto FR, Andrade TG. Use of EuroSCORE as a predictor of morbidity after cardiac surgery. Rev Bras Cir Cardiovasc. 2014;29(1):9-15.

15. Roques F, Nashef SA, Michel P, Gauducheau E, Vincentiis C, Baudet E, et al. Risk factors and outcome in European cardiac surgery: analysis of the EuroSCORE multinational database of 19030 patients. Eur J Cardiothorac Surg. 1999;15(6):816-22.

16. Knaus WA, Draper EA, Wagner DP, Zimmerman JE. APACHE II: a severity of disease classification system. Crit Care Med. 1985;13(10):818-29.

17. Le Gall JR, Lemeshow S, Saulnier F. A new Simplified Acute Physiology Score (SAPS II) based on a European/North American multicenter study". JAMA. 1993;270(24):2957-63.

18. VincentJL, Moreno R, Takala J, Willatts S, De Mendonça A, Bruining H, et al. The SOFA (Sepsis-related Organ Failure Assessment) score to describe organ dysfunction/failure. On behalf of the Working Group on Sepsis-Related Problems of the European Society of Intensive Care Medicine. Intensive Care Med. 1996;22(7):707-10.

19. Canver CC, Chanda J. Intraoperative and postoperative risk factors for respiratory failure after coronary bypass. Ann Thorac Surg. $2003 ; 75(3): 853-7$.

20. Hosseinian L, Chiang Y, Itagaki S, Polanco A, Rhee A, Chikwe J. Earlier versus later tracheostomy in patients with respiratory failure after cardiac surgery in the United States. J Cardiothorac Vasc Anesth. 2014;28(3):488-92.

21. Brown $\mathrm{CH}$. Delirium in the cardiac surgical ICU. Curr Opin Anaesthesiol. $2014 ; 27(2): 117-22$

22. Jung P, Pereira MA, Hiebert B, Song X, Rockwood K, Tangri N, et al. The impact of frailty on postoperative delirium in cardiac surgery patients. J Thorac Cardiovasc Surg. 2015;149(3):869-75.

23. Rudolph JL, Inouye SK, Jones RN, Yang FM, Fong TG, Levkoff Se, et al. Delirium: An independent predictor of functional decline after cardiac surgery. J Am Geriatr Soc. 2010;58(4):643-9.

24. McPherson JA, Wagner CE, Boehm LM, Hall JD, Johnson DC, Miller LR, et al. Delirium in the cardiovascular ICU: exploring modifiable risk factors. Crit Care Med. 2013;41(2):405-13. 
25. Cavallazzi R, Saad M, Marik PE. Delirium in the ICU: an overview. Ann Intensive Care. 2012;2(1):49-60

26. Huen SC, Parikh CR. Predicting acute kidney injury after cardiac surgery: a systematic review. Ann Thorac Surg. 2012;93(1):337-47.

27. Yoon JC, Kim YJ, Lee YJ, Ryoo SM, Sohn CH, Seo Dw, et al. Serial evaluation of SOFA and APACHE II scores to predict neurologic outcomes of out-of-hospital cardiac arrest survivors with targeted temperature management. PloS One. 2018;13(4):1-11.
28. Saxena A, Meshram SV. Predictors of mortality in acute kidney injury patients admitted to Medicine Intensive Care Unit in a rural tertiary care hospital. Indian J Crit Care Med. 2018;22(4):231-7.

29. Rapsang AG, Shyam DC. Scoring System in the intensive care unit: a compendium. Indian J Crit Care Med. 2014;18(4):220-8.

30. Ferreira FL, Bota DP, Bross A, Mélot C, Vincent JL. Serial evaluation of the SOFA score to predict outcome in critically ill patients. JAMA $2001 ; 286(14): 1754-8$. 